\title{
Coherent control of light field with electromagnetically induced transparency in a dark state Raman coherent tripod system
}

\author{
YongYao Li ${ }^{1,2}$, HuaRong Zhang ${ }^{1}$, YongZhu Chen ${ }^{1,3}$, and JianYing Zhou周 \\ ${ }^{1}$ State Key Laboratory of Optoelectronic Materials and Technologies, \\ Sun Yat-sen University, Guangzhou 510275, China \\ ${ }^{2}$ Department of Applied Physics,South China Agricultural University, Guangzhou 510642, China \\ ${ }^{3}$ School of Electro-Mechanic Engineering, Guangdong Polytechnic Normal University, Guangzhou 510665, China
}

(Dated: April 23, 2022)

\begin{abstract}
The coherent superposition of two-atomic levels induced by coherent population trapping is employed in a standard $\Lambda$ type scheme to form a tripod-like system. A weak probe pulse scanning across the system is shown to experience a crossover from absorption to transparent and then to amplifcation. Consequently the group velocity of the probe pulse can be controlled to propagate either as a subluminal, a standard, a superluminal or even a negative speed. It is shown that the propagation behavior of the light field is entirely determined and controlled by the initial states of the coherent superposition.
\end{abstract}

PACS numbers: Valid PACS appear here

\section{INTRODUCTION}

Coherent population trapping (CPT) [1, 2, 3] and Electromagnetically induced transparency (EIT) [4, 5] are two different quantum coherence processes which have led to the observation of many new physics effects in quantum optics and atomic physics. CPT is a preparation of atoms in a coherent superposition of ground and metastable state sublevels, which is called dark state, for the reason that this state is immune to excitation by a two-component laser radiation under the two-photon resonance condition. EIT can also be classified as a dark state. It can modify the optical properties of a medium and results in making a resonant opaque medium transparent, which has been observed in a wide range of atomic, molecular, and condensed matter systems [6, 7, [8]. In the past two decades, potential applications of EIT to coherent control for group velocities of the probe field in quantum information and quantum communication [9, 10, 11, 12], to left-handed material for negative refraction [13, 14, 15] and to enhanced nonlinear optics for frequency conversion [16, 17] have been reported. The distinct feature of EIT and CPT is that EIT causes not only a modification of optical property of the medium but also the optical fields themselves.

In this paper, we analyse the optical property of a fivelevel atomic configuration illustrated in FIG. 1. Two coherent fields with complex Rabi-frequencies $\Omega_{1}$ and $\Omega_{2}$ create a dark state superposition of states $|1\rangle$ and $|2\rangle[1]$. By introducing a coherent superprosition in the system, more degrees of freedom to the system can be added, then we will gain a control over more physical variables [18, 19]. The coherent superprosition of the dark state is given as

*Electronic address: stszjy@mail.sysu.edu.cn below:

$$
|D\rangle=\cos \theta|1\rangle-\sin \theta|2\rangle
$$

Here

$$
\begin{aligned}
& \cos \theta=\frac{\Omega_{1}}{\sqrt{\Omega_{1}^{2}+\Omega_{2}^{2}}} \\
& \sin \theta=\frac{\Omega_{2}}{\sqrt{\Omega_{1}^{2}+\Omega_{2}^{2}}}
\end{aligned}
$$

By scaning a weak probe field through the system, the system exhibits a rich optical properties under different initial conditions because of the Raman coherence of the dark state. We found that not only double EIT channels [20] and left-handed material 15] but also a lot of other effects such as Raman amplification 21], refractive index enhancement 22], slow light 9, 10] and superluminal light [23] can be simply achieved by adiabatically [24, 25] changing the initial state.

The mixing angle $\theta$ in Eq. (2) is determined by the relative intensity of $\Omega_{1}$ and $\Omega_{2}$. This dark state plays the role of the original ground state. Here the system resembles a three-level $\Lambda$-type system. The probe field then experiences a transition from the dark state $|D\rangle$ to the upper state $|4\rangle$. A strong resonant coherent field $\Omega_{C}$ is employed to couple states $|3\rangle$ and $|4\rangle$.

The technique to establish the coherent preparation of a dark state was realized in many systems [1]. In our case, the atoms are trapped in state $|1\rangle$ and state $|2\rangle$ with the probability of $\cos ^{2} \theta$ and $\sin ^{2} \theta$ respectively. The probability is decided by the mixing angle $\theta$, which can be tuned adiabatically by changing the relative intensity of $\Omega_{1}$ and $\Omega_{2}$. Because none of the atoms is prepared at the state $|e\rangle$ and the probe field is weak, the contribution from level $|e\rangle$ to the system can be neglected safely. The energy levels $(|1\rangle,|2\rangle,|3\rangle$ and $|4\rangle)$ can be viewed as a tripod level configuration [25, 26, 27, 28, 29]. . 


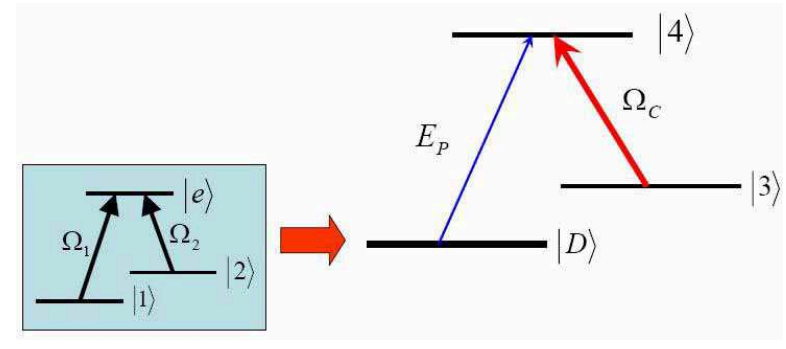

FIG. 1: Energy-Level scheme of the system: two coherent laser field with Rebi-frequency $\Omega_{1}$ and $\Omega_{2}$ coupling of levels $|1\rangle,|2\rangle$ and $|e\rangle$ create the dark state $|D\rangle$, which construct a $\Lambda$ type scheme with $|3\rangle$ and $|4\rangle$. A probe field $E$ transits from dark state $|D\rangle$ to the upper level $|4\rangle$ and a coupling field transits with $|3\rangle$ and $|4\rangle$

\section{EQUATIONS AND SOLUTIONS}

The Hamiltonian of this four-level system can be obtained in the rotating frame by introducing the rotatingwave approximation:

$$
\begin{aligned}
H= & \sum_{i=1}^{4} \hbar \omega_{i} \sigma_{i i}-\frac{\hbar}{2}\left[\Omega_{1 P} e^{-i \nu_{P} t} \sigma_{41}+\Omega_{2 P} e^{-i \nu_{P} t} \sigma_{42}\right. \\
& \left.+\Omega_{C} e^{-i \nu_{C} t} \sigma_{43}+H . C\right]
\end{aligned}
$$

Here $\omega_{i}$ is the eigenfrequency of the energy level and $\sigma_{i j}=|i\rangle\langle j|$, Rabi-frequency $\Omega_{1 P}=\wp_{41} \mathscr{E}_{P} / \hbar, \Omega_{2 P}=$ $\wp_{41} \mathscr{E}_{P} / \hbar$ and $\Omega_{C}=\wp_{43} \mathscr{E}_{C} / \hbar$, where $\wp_{i j}$ is the electric dipole moment from $|i\rangle$ to $|j\rangle, \mathscr{E}_{P}$ and $\mathscr{E}_{C}$ is the slowly varying amplitude of the probe field and the coupling field respectively. Assume that the electric dipole moment $\wp_{41} \approx \wp_{42}=\wp$ and the value of $\wp$ is real, which results that $\Omega_{1 P} \approx \Omega_{2 P}=\Omega_{P}$.

The evolution equation of density matrix reads:

$$
\dot{\rho}=-\frac{i}{\hbar}[H, \rho]-[\Gamma, \rho]_{+}
$$

The matrix element $\langle i|\Gamma| j\rangle=\gamma_{i} \delta_{i j}$, where $\gamma_{i}$ is the decay rate designating the population damping from the energy level $|i\rangle$. To simplify, we assume that $\Omega_{1}$ and $\Omega_{2}$ are real, and $\gamma_{4} \gg \gamma_{3} \approx \gamma_{2} \approx \gamma_{1} \approx 0$. With these approximations, the population of the atoms are prepared at the energy levels $|1\rangle,|2\rangle$ and $|3\rangle$ and the atoms are initially set to:

$$
\begin{aligned}
& \rho_{11}^{(0)}=\cos ^{2} \theta, \rho_{22}^{(0)}=\sin ^{2} \theta \\
& \rho_{21}^{(0)}=\rho_{12}^{(0)}=-\cos \theta \sin \theta
\end{aligned}
$$

The initial conditions indicate that $\cos ^{2} \theta$ and $\sin ^{2} \theta$ denotes population of the atoms prepared at the energy level $|1\rangle$ and $|2\rangle$ respectively. While $-\cos \theta \sin \theta$ denotes the dark state Raman coherence between levels $|1\rangle$ and $|2\rangle$.

The equations of density matrix elements in slowly varying amplitude approximation are:

$$
\begin{aligned}
& \dot{\rho}_{41}=-\left(i \Delta_{1}+\gamma_{41}\right) \rho_{41}+\frac{i}{2} \Omega_{P} \rho_{11}+\frac{i}{2} \Omega_{P} \rho_{21}+\frac{i}{2} \Omega_{C} \rho_{31} \\
& \dot{\rho}_{31}=-i\left(\Delta_{1}-\Delta_{C}\right) \rho_{31}+\frac{i}{2} \Omega_{C}^{*} \rho_{41} \\
& \dot{\rho}_{42}=-\left(i \Delta_{2}+\gamma_{42}\right) \rho_{42}+\frac{i}{2} \Omega_{P} \rho_{22}+\frac{i}{2} \Omega_{P} \rho_{12}+\frac{i}{2} \Omega_{C} \rho_{32} \\
& \dot{\rho}_{32}=-i\left(\Delta_{2}-\triangle_{C}\right) \rho_{32}+\frac{i}{2} \Omega_{C}^{*} \rho_{42}
\end{aligned}
$$

The off-diagonal decay rates $\gamma_{i j}$ are given by $\gamma_{i j}=$ $\left(\gamma_{i}+\gamma_{j}\right) / 2$ denoting the total coherence relaxation rates between states $|i\rangle$ and $|j\rangle$. The detuings are defined as $\triangle_{1}=\omega_{41}-\nu_{P}, \Delta_{2}=\omega_{42}-\nu_{P}, \triangle_{C}=\omega_{43}-\nu_{C}$. As shown in the system, one finds that:

$$
\Delta_{1}-\Delta_{2}=\omega_{21}
$$

The steady-state solutions of $\rho_{41}$ and $\rho_{42}$ are given by:

$$
\begin{gathered}
\rho_{41}=\frac{\frac{i}{2} \Omega_{P}\left(\cos ^{2} \theta-\cos \theta \sin \theta\right)\left(\triangle_{1}-\triangle_{C}\right)}{\left(i \triangle_{1}+\gamma_{41}\right)\left(\triangle_{1}-\triangle_{C}\right)-i \Omega_{C}^{2} / 4} \\
\rho_{42}=\frac{\frac{i}{2} \Omega_{P}\left(\sin ^{2} \theta-\cos \theta \sin \theta\right)\left(\triangle_{2}-\triangle_{C}\right)}{\left(i \triangle_{2}+\gamma_{42}\right)\left(\triangle_{2}-\triangle_{C}\right)-i \Omega_{C}^{2} / 4}
\end{gathered}
$$

The polarization $\mathscr{P}$ in the slowly varying frame and the susceptibility $\chi$ are related to each other with the equation [30]:

$$
\mathscr{P}=\epsilon_{0} \chi(\omega) \mathscr{E}_{P}=2 N\left(\wp_{14} \rho_{41}+\wp_{24} \rho_{42}\right)
$$

where $N$ is the total number of atoms.

The expression of the susceptibility is given as below:

$$
\begin{aligned}
\chi(\omega, \theta)=\quad & i K\left[\frac{f(\theta)\left(\Delta_{1}-\Delta_{C}\right)}{\left(i \Delta_{1}+\gamma_{41}\right)\left(\triangle_{1}-\triangle_{C}\right)-i \Omega_{C}^{2} / 4}\right. \\
& +\frac{g(\theta)\left(\Delta_{2}-\triangle_{C}\right)}{\left(i \Delta_{2}+\gamma_{42}\right)\left(\triangle_{2}-\triangle_{C}\right)-i \Omega_{C}^{2} / 4}
\end{aligned}
$$

Where $K=N \wp^{2} / \epsilon_{0} \hbar$, and $f(\theta)=\cos ^{2} \theta-\cos \theta \sin \theta$, $g(\theta)=\sin ^{2} \theta-\cos \theta \sin \theta$

Assume that $\gamma_{41} \approx \gamma_{42}=\gamma$ and $\Delta_{C}=0$, the real part and imaginary part of susceptibility are given as below:

$$
\begin{aligned}
R e[\chi]= & K\left[f(\theta) \frac{\triangle_{1}\left(\triangle_{1}^{2}-\Omega_{C}^{2} / 4\right)}{\gamma^{2} \triangle_{1}^{2}+\left(\triangle_{1}^{2}-\Omega_{C}^{2} / 4\right)^{2}}\right. \\
& \left.+g(\theta) \frac{\triangle_{2}\left(\triangle_{2}^{2}-\Omega_{C}^{2} / 4\right)}{\gamma^{2} \triangle_{2}^{2}+\left(\triangle_{2}^{2}-\Omega_{C}^{2} / 4\right)^{2}}\right] \\
\operatorname{Im}[\chi]= & K\left[f(\theta) \frac{\gamma \triangle_{1}^{2}}{\gamma^{2} \triangle_{1}^{2}+\left(\triangle_{1}^{2}-\Omega_{C}^{2} / 4\right)^{2}}\right. \\
& \left.+g(\theta) \frac{\gamma \triangle_{2}^{2}}{\gamma^{2} \triangle_{2}^{2}+\left(\triangle_{2}^{2}-\Omega_{C}^{2} / 4\right)^{2}}\right]
\end{aligned}
$$




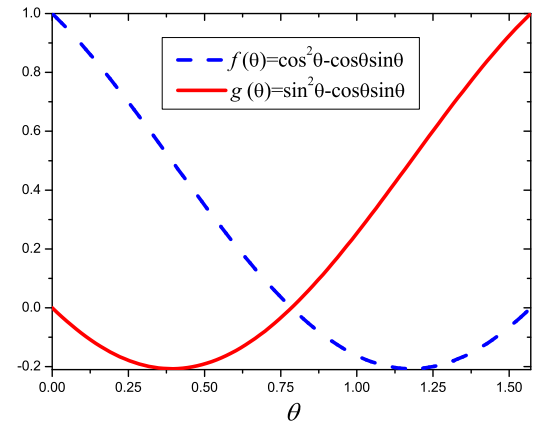

FIG. 2: Function of $f(\theta)$ (solid line) and $g(\theta)$ (dash line) in $[0, \pi / 2]$ the minimum of the two expression appear at $\theta=\pi / 8$ and $\theta=3 \pi / 8$

\section{ANALYSIS OF THE SOLUTION}

Eq. (10) and Eq. (11) show that the susceptibility is mainly determined by $f(\theta)$ and $g(\theta) . f(\theta)$ affects the dispersion relationship in the vicinity of the resonance with $\Delta_{1}=0$ and $g(\theta)$ affects the relationship near the resonance at $\Delta_{2}=0$. The term of $\cos ^{2} \theta$ and $\sin ^{2} \theta$ in $f(\theta)$ and $g(\theta)$, denotes the population of the atoms at the energy level $|1\rangle$ or $|2\rangle$ respectively and relates with the absorption to the probe field. While the term of $-\cos \theta \sin \theta$ denotes the dark state Raman coherence between $|1\rangle$ and $|2\rangle$ and gives rise Raman amplification to the probe field. The behavior of these two functions at the range of $[0, \pi / 2]$ are plotted in FIG. 2.

It is shown from FIG. 2 that $f(\theta)>0$ and $g(\theta)<0$ for $\theta<\pi / 4$. This indicates that the probe experiences absorption in the vicinity of $\Delta_{1}=0$ and gain in the vicinity of $\Delta_{2}=0$. For $\theta>\pi / 4$, on the other hand, the probe experiences gain in the vicinity of $\Delta_{1}=0$ and absorption in the vicinity of $\Delta_{2}=0$. Therefor, the dark state superposition creates mixtures of active and passive optical materials at frequencies near their resonances [31].

Particularly, when the mixing angle $\theta=\pi / 4$, we have $\chi(\omega)=0$. In this case, both the real part and the imaginary part of susceptibility are vanished. Raman coherence of the dark states induces transparency at all range of frequency, and the probe field propagates in the medium just as it propagates through the vacuum.

The minimum of the two functions appear at $\theta=\pi / 8$ and $\theta=3 \pi / 8$, so that the max gain in vicinity of two resonances is appearing respectively correspond to $\theta$ at these points. We plot the real part and the imaginary part of the susceptibility in FIG. 3. The special points of $\theta=\pi / 8$ and $\theta=3 \pi / 8$ are also plotted respectively in FIG. 3.

FIG. 3 (a) and (b) show that the population is trapped in level $|1\rangle$ for $\theta=0$. In the vicinity of $\Delta_{1}=0$, the real and the imaginary part of the susceptibility exhibit a typical EIT phenomenon in a standard $\Lambda$ system. Similarly, for the case of $\theta=\pi / 2$, the population is trapped

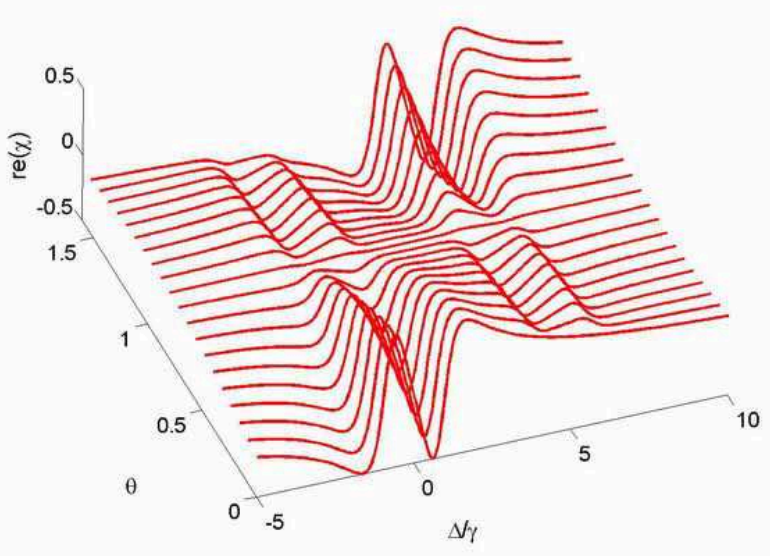

(a)

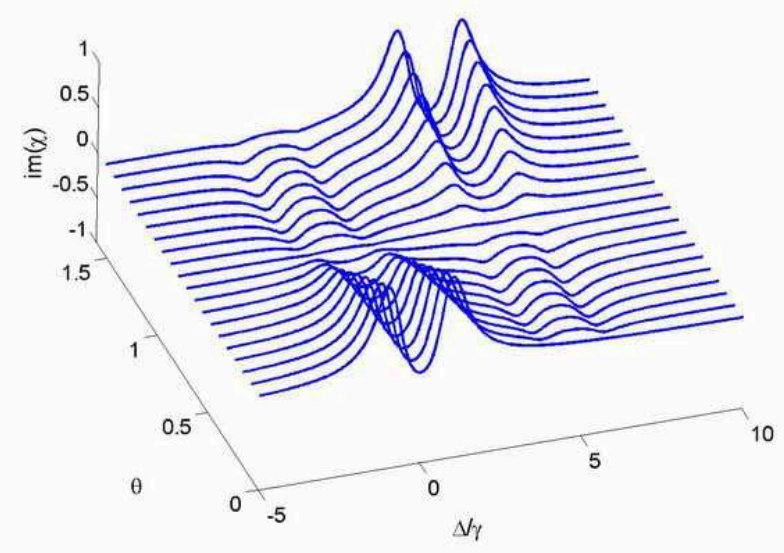

(b)

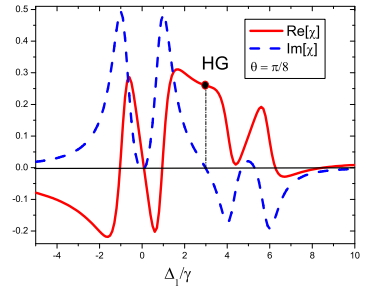

(c)

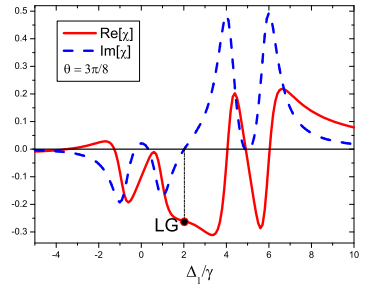

(d)
FIG. 3: Real and imaginary parts of the susceptibility as a function of the probe field detuning $\Delta_{1} / \gamma$ and the mixing angle $\theta$. We assume that the material is cold atomic gas with $N \sim 1.0 \times 10^{12} \mathrm{~cm}^{-3}, \gamma \sim 10 \mathrm{MHz}, \Omega_{C} \approx 2 \gamma, \omega_{21} \approx 5 \gamma$ and $K \approx \gamma$. (a) and (b) 3D graph about the real part and the imaginary of the susceptibility. The point of 0 at the axes of $\Delta / \gamma$ is related to the resonance of $\Delta_{1}=0$ and the point of 5 is relate to the resonance of $\triangle_{2}=0$. (c) and (d) Dispersion relationship when $\theta=\pi / 8$ and $\theta=3 \pi / 8$. ' $H G$ ' is a high refractive index without absorption and ' $L G$ ' is a negative refractive index without absorption. 


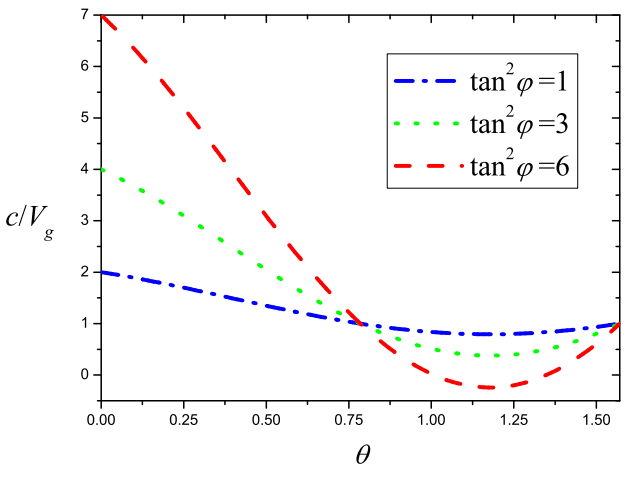

FIG. 4: Group velocity as a function of $\theta(\theta \in[0, \pi / 2])$ in some different mixing angle of $\varphi$

in level $|2\rangle$ and a typical EIT phenomenon is appeared near $\Delta_{2}=0$. When the mixing angle $\theta$ increases from 0 to $\pi / 2$, the real part of the susceptibility in vicinity of resonance at $\Delta_{1}=0$ changes from normal dispersion to abnormal dispersion and contrary similar process can be observed in vicinity of $\triangle_{2}=0$. As the imaginary parts of the susceptibility near this two resonances are varied proportional to the function of $f(\theta)$ and $g(\theta)$ respectively. Especially, one can find that both the real part and the imaginary part of the susceptibility are viewed as a straight line in these two figures for the case of $\theta=\pi / 4$.

FIG. 3 (c) and (d) show that two points which is labeled as 'HG' and 'LG' . The point of 'HG' has an high refractive index with zero absorption at $\theta=\pi / 8$, while the 'LG' has an negative refractive index with zero absorption at $\theta=3 \pi / 8$.

Assuming a plus with Rabi-frequency $\Omega_{P}(z, t) e^{-i \nu t}$ propagation in this medium. The Maxwell-Bloch equation in one-dimensional slowly-varying envelope approximation is [30]:

$$
\left(\frac{\partial}{\partial t}+c \frac{\partial}{\partial z}\right) \Omega_{P}(z, t)=-\frac{\omega_{41} \wp}{2 \epsilon_{0} \hbar} \operatorname{Im}\left(2 N \rho_{41}+2 N \rho_{42}\right)
$$

Let the carrier frequency of the plus $\nu=\omega_{41}$, the effect of the $N \rho_{42}$ on the right hand side of the Eq. (13) can be neglected. With the approximation as that adopted in Ref [12], one can obtain that:

$$
\begin{aligned}
& \rho_{41}=-i \frac{2}{\Omega_{C}} \frac{\partial}{\partial t} \rho_{31} \\
& \rho_{31} \approx-\frac{\Omega_{P}(z, t)}{\Omega_{C}} f(\theta)
\end{aligned}
$$

We assume that $\Omega_{C}$ is real and constant, then the propagation equation is changed to:

$$
\left(\frac{\partial}{\partial t}+c \frac{\partial}{\partial z}\right) \Omega_{P}(z, t)=-\frac{g^{2} N}{\Omega_{C}^{2}} \frac{\partial}{\partial t} \Omega_{P}(z, t) f(\theta)
$$

Here $g=\wp \sqrt{2 \omega_{41} / \epsilon_{0} \hbar}$, and we define that $\tan ^{2} \varphi=$ $g^{2} N / \Omega_{C}^{2}$. If the mixing angle $\theta$ is not variable as a function of time. Eq. (15) can be simplified to:

$$
\left(\frac{\partial}{\partial t}+V_{g} \frac{\partial}{\partial z}\right) \Omega_{P}(z, t)=0
$$

And

$$
V_{g}=\frac{c}{1+f(\theta) \tan ^{2} \varphi}
$$

It is interesting to compare Eq. (17) to the standard expression of group velocity in the dispersion media given by:

$$
V_{g}=\frac{c}{n(\omega)+\omega \frac{d n}{d \omega}}
$$

Because the carrier frequency which the light field in near resonance with level $|1\rangle$ and $|4\rangle$, according to the property of the EIT, the refractive index of the light field $\left.n(\omega)\right|_{\triangle_{1}=0}=\sqrt{1+\left.\operatorname{Re}\{\chi(\omega)\}\right|_{\triangle_{1}=0}} \approx 1$. The comparision shows $\left.\omega\left(\frac{d n}{d \omega}\right)\right|_{\Delta_{1}=0} \sim f(\theta) \tan ^{2} \varphi$. Then we can draw a conclusion that the sign of $f(\theta)$ determines the light pulse propagate in the medium for a normal dispersion or an abnormal dispersion.

For example, at $\theta>\pi / 4$, and with near resonant excitation at $\Delta_{1}=0$, we have the normal dispersion. However, when $\theta>\pi / 4$, it is an abnormal dispersion with $f(\theta)<0$, leading to a superluminal light pulse [23]. FIG. 4 shows that the group velocity as a function of $\theta$ in some of the mixing angle $\varphi$.

Especially, when

$$
\tan ^{2} \varphi>\left|\frac{1}{f_{\min }(\theta)}\right| \approx 4.83
$$

the group velocity can become negative. So that one can achieve a desirable group velocity simply by setting different mixing angle $\theta$ and $\varphi$ in this system.

An interesting extension of the topic is to vary $\Omega_{1}$ or $\Omega_{2}$ in the space 32,33 . This treating leads to a spatially dependent population distribution at levels $|1\rangle$ and $|2\rangle$ and hence a spatially dependent of the dispersion relation. If the variation is periodic in the space, it can produce a photonic band gap (PBG) structure in the medium [34, 35].

\section{CONCLUSIONS}

Controlled group velocity of light in the tripod system with EIT is an interesting topic which leads to rich phenomena [36]. In this contribution, coherent superposition of a dark state is proposed in a four-level tripod system to control the probe propagation through EIT in the medium. It is found that the dark state superposition creates mixtures of active and passive optical materials at frequencies near their resonances and leading to a 


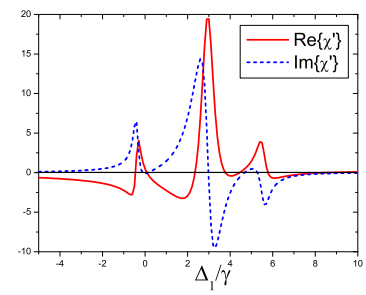

(a)

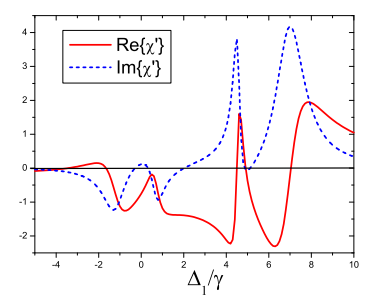

(b)
FIG. 5: Real and imaginary parts of the susceptibility in the local field corrections. We chose $N \sim 1.0 \times 10^{13} \mathrm{~cm}^{-3}, \gamma \sim$ $10 \mathrm{MHz}, \Omega_{C} \approx 2 \gamma, \omega_{21} \approx 5 \gamma$ and $K \approx 10 \gamma$. (a) and (b): Dispersion relationship in the local field corrections with $\theta=$ $\pi / 8$ and $\theta=3 \pi / 8$.

controllable the group velocity either as a sublimunal, a standard, a superlimunal or even a negative speed. And the propagation behavior is entirely determined by the mixing angle $\theta$ and $\varphi$ of the initial states.

The energy levels in the present paper can be established in a wide range of atomic, molecular, and condensed matter. Rb vapor, metastable neon, rareearth atoms, donorbound electrons and bound excitons in semiconductors [9, 37, 38, 39, 40] are appropriate sample because these medium have abundant level structures. Ref. 15] gives an example 41] of this system in practice. If increasing the density of the material, the local field effect [42] must take into account. The electric field replaces to the microscopic field $E_{m}=E+P / 3 \epsilon_{0}$ and the dielectric constant is changed as below:

$$
\epsilon-1=\frac{N \alpha}{1-N \alpha / 3}
$$

where $\alpha$ is the total polarizability. FIG. 5 plots the susceptibility with $\theta=\pi / 8$ and $\theta=3 \pi / 8$ in the local field correction.

\section{Acknowledgments}

YongYao Li thanks Dr JingFeng Liu \& GuiHua Chen and Prof WeiMin Kuang \& Jing Cheng for useful discussion. HuaRong Zhang thanks for the advice from Prof XiangYang Yu. This work is supported by the National Key Basic Research Special Foundation (NKBRSF) (G2004CB719805), Chinese National Natural Science Foundation $(60677051,10774193)$.
[1] K. Bergmann, H. Theuer, W. Shore, Rev. Mod. Phys. 70, 1003(1998).

[2] M. O. Scully, Phys. Rev. Lett. 67, 1855(1991).

[3] R. M. Whitley, and C. R. Stoud. Jr, Phys. Rev. A 14, 1498(1976).

[4] S. E. Harris, Phys. Today 50, No.7, 36(1997).

[5] M. Fleischhauer, A. Imamoglu, and J. P. Marangos, Rev. Mod. Phys. 77, 633-673 (2005).

[6] K. J. Boller, A. Imamoglu, and S. E. Harris, Phys. Rev. Lett. 66, 2593(1990).

[7] B. S. Ham, P. R. Hemmer, M. S. Shahriar, Opt. Commun. 144, 227(1997).

[8] G. B. Serapiglia, E. Paspalakis, et.al, Phys. Rev. Lett. 84, 1019 (2000).

[9] M. Xiao, Y. Q. Li, S. Z. Jin, and J. Gea-Banacloche, Phys. Rev. Lett. 74, 666(1995).

[10] L. V. Hau, S. E. Harris, Z. Dutton, and C. H. Behroozi, Nature(London). 397, 594(1999).

[11] O. Kocharovskaya, Y. Rostovtsev, and M. O. Scully, Phys. Rev. Lett. 86, 628(2001).

[12] M. Fleischhauer, and M. D. Lukin, Phys. Rev. Lett. 84, 5094(2000). M. Fleischhauer, and M. D. Lukin, Phys.Rev.A 65, 022314(2002). M. D. Lukin, Rev. Mod. Phys. 75, 457-472(2003).

[13] M. Ö. Oketel and M. Ö. Mstecaplioglu, Phys. Rev. A 70, 053806(2004).

[14] Q. Thommen and P. Mandel, Phys. Rev. Lett. 96, 053601(2006).

[15] J. Kästel, M. Fleischhauer, S. F. Yelin, and R. L. Walsworth, Phys.Rev.Lett. 99, 073602(2007).

[16] S. E. Harris, J. E. Field, and A. Imamoglu , Phys. Rev.
Lett. 77, 1107(1990).

[17] S. E. Harris, and Y. Yamamoto, Phys. Rev. Lett. 81, 3611(1998).

[18] G. Kuriziki and M.Shapiro, Phys. Rev. B 39, 3435(1988).

[19] E. Paspalakis, and P. L. Knight, J. Mod. Opt. 49, 8795(2002)

[20] S. M. Iftiquar, arXiv:0711:2232v1 (2007).

[21] R. Kolesov, Phys. Rev. A 64, 063819(2001).

[22] M. O. Scully, Phys. Rev. Lett. 67, 1855(1991).

[23] L. J. Wang, A. Kuzmich, A. Dogariu, Nature(London), 406, 277(2001).

[24] J. Oreg, F. T. Hioe, J. H. Eberly, Phys. Rev. A 29, 690(1984).

[25] Z. Kis, and F. Renzoni, Phys. Rev. A 65, 032318(2002).

[26] B. S. Ham, P. R. Hemmer, Phys. Rev. Lett. 84, 4081(2000).

[27] I. E. Mazets, Phys. Rev. A 71, 023806(2005).

[28] C. Goren, A. D. Wilson-Gordon, M. Rosenbluh, and H. Friedmann, Phys. Rev. A 69, 063802(2004).

[29] D. Petrosyan, Y. P. Malakyan, Phys. Rev. A 70, 023822(2004).

[30] M. O. Scully, and S. Zubairy, Quantum Optics (Cambridge University, Cambridge, England,1997).

[31] V. Anant, A. F. Abouraddy, and K. K. Berggren arXiv:0711.5021 1 (2007)

[32] M. Bajcsy, A. S. Zibrov, and M. D. Lukin, Nuture(Lodon), 426, 638(2003).

[33] X. S. Xie, M. Li, et.al. Opt. Express. 15, 7023(2007).

[34] A. André, and M. D. Lukin, Phys. Rev. Lett. 90, 143602(2002).

[35] QiongYi He, Xue Yan et.al. Phys. Rev. B 73, 
195124(2006).

[36] E. Paspalakis, N. J. Kylstra, and P. L. Knight, Phys. Rev. A 65, 053808(2002). E. Paspalakis, and P. L. Knight, Phys. Rev. A 66, 015802(2002). E. Paspalakis, and Z. Kis, Phys. Rev. A 66 , 025802(2002).

[37] Y. C. Chen, C. W. Lin et.al. Phys. Rev. A 61, 053805(2000).

[38] H. F. Zhang, J. H. Wu, X. M. Su and J. Y. Gao, Phys.
Rev. A 66, 053816(2002)

[39] Y. Z. Wang, G. Xu,et.al. Phys. Rev. A 53, 1160(1996).

[40] Y. Zhao, C. Wu, B. S. Ham, et.al. Phys.Rev.Lett. 79, 641(1997)

[41] A. J. Flikweert, et.al. J. Appl. Phys. 98, 073301(2005).

[42] N. Wiser Phys. Rev, 129, 62(1962). C. M. Bowden and J. P. Dowling, Phys. Rev. A 47, 1247(1993). 